\title{
DIRECTIONAL PROPAGATION CANCELLATION FOR ACOUSTIC COMMUNICATION ALONG THE DRILL STRING
}

Sinan Sinanović, Don H. Johnson

\author{
Computer and Information Tech. Inst. \\ Dept. of Elec. and Comp. Eng. \\ Rice University \\ Houston, Texas 77251-1892 \\ sinanerice.edu, dhjerice.edu
}

\author{
Wallace R. Gardner \\ Halliburton Energy Services \\ 3000 N Sam Houston Pkwy E \\ P.O. Box 60078 (77205) \\ Houston, TX 77479 \\ wally.gardnerehalliburton.com
}

\begin{abstract}
A new telemetry method in oil well services uses compressional acoustic waves to transmit data along the drill string to the surface. Normal drilling operations produce in-band acoustic noise at intensities comparable to the transducer output while lossy propagation through the drill string and surface noise further degrade the signal. A single receiver system has a capacity of several hundreds bits per second. A two-receiver scheme exploits the fact that the surface noise source and the signal propagate in opposite directions to remove the downward propagating surface noise, which produces substantial increases in channel capacity. We use training with easily obtained data to determine how the signals need to be processed in a way that does not rely on knowing sensor placement or the acoustic model.
\end{abstract}

\section{INTRODUCTION}

The success in finding the oil reserves depends, in part, on real-time (while-drilling) information acquired by multiple sensors placed close to the drill bit. This information, once transmitted to the surface, can be used to optimize the drilling by adjusting the direction of drilling or determining the proximity of oil reserves in the formation. Currently, two telemetry methods are used: wireline telemetry and mud-pulse telemetry. In wireline telemetry, the measurements are converted into electrical signals and sent up a coaxial cable. Wireline telemetry provides a large capacity but interferes with drilling operations. Alternatively, mudpulse telemetry, a traditional telemetry method, can be used while drilling but it achieves very low data rates, typically less than $10 \mathrm{bits} / \mathrm{s}$ and provides only one way communication: from the drill bit to the surface [1].

A new method of wireless data telemetry uses compressional acoustic waves to transmit data up and down the drill

This research was supported by Halliburton Energy Services. string. Many physical constraints present challenges for this type of telemetry. Acoustic wave propagation through the drill string encounters attenuation and scattering due to the acoustic impedance mismatch at the pipe joints which results in a lossy, non-flat transfer function [1]. Furthermore, downhole and surface noise sources produce substantial inband power, resulting in low signal-to-noise ratio. This paper describes a way of improving the channel capacity by using two spatially separated receivers (instead of one) on the drill string protruding above the surface.

\section{MODEL FOR THE ACOUSTIC COMMUNICATION IN THE DRILL STRING}

Normal drilling operations produce in-band acoustic noise at multiple sources at intensities comparable to the transducer output. During the drilling, the drill bit crushes the formation and creates compressional acoustic waves that propagate in the drill string. Since the drill string consists of many pipe segments, compressional acoustic waves also partly reflect at the pipe joints, resulting in a multiband transfer function. Finally, surface noise, the result of the surface drilling operations, further degrades the signal sent by the transducer. We use the idealized model as depicted on the Figure 1 to analyze the performance of acoustic telemetry. The model is assumed to be linear, as in [1].

\section{CAPACITY IMPLICATIONS}

We consider here the case of uplink telemetry signal $s(t)$ contaminated by the bit noise $n_{b}(t)$ and transmitted to the surface. The following notation is used: $P_{N_{b}}(f)$ denotes the bit noise PSD (power spectral density), $P_{N_{s}}(f)$ the surface noise PSD, $P_{S}(f)$ the signal PSD and $H(f)$ denotes the drill string frequency response (transfer function). For a drill string of several thousand feet of length, $H(f)$ exhibits 


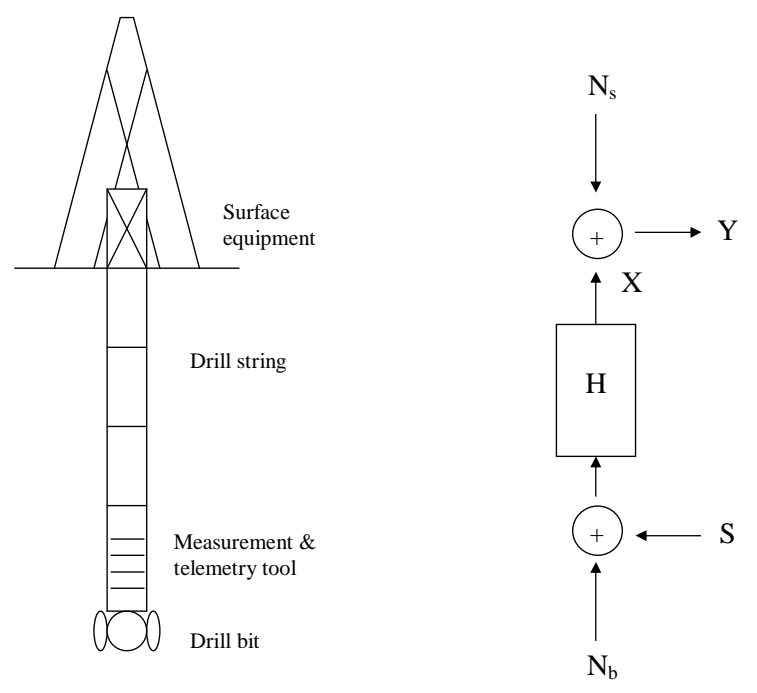

Fig. 1. The figure to the left depicts a simplified oil rig model with a derrick on the surface and the drill string, composed of jointed pipes, stretching from the surface down to the drill bit. The figure to the right shows idealized oil rig system model used in analysis of the acoustic telemetry data rates. $S$ denotes the telemetry signal's spectrum, $Y$ denotes received signal's spectrum, $N_{b}$ denotes the bit noise spectrum, $N_{s}$ denotes the surface noise spectrum and $H$ denotes the uplink drill string transfer function.

small gains $(|H(f)| \ll 1)$ with many passbands and stopbands due to the multiple reflections of the acoustic waves in the drill string. Furthermore, attenuation even in the passbands becomes more severe as the length of the drill string increases. We assume both noise sources to be additive and Gaussian. The expression for the uplink capacity over the frequency band $B$ is given by $[3,4]$ :

$C_{U L}=\int_{B} \log _{2}\left(1+\frac{P_{S}(f)}{P_{N_{b}}(f)+P_{N_{s}}(f)|H(f)|^{-2}}\right) d f$ bits/s

For a given bandwidth $B$, the capacity depends on the SNRlike quantity inside the logarithm. The denominator inside the logarithm shows that the surface noise is, in effect, greatly amplified by $|H(f)|^{-2}$ because $|H(f)|^{-2} \gg 1$. Therefore, if $P_{N_{b}}(f)$ and $P_{N_{s}}(f)$ are of the same order of magnitude, the capacity formula shows that the dominant noise component in the capacity formula is the surface noise because of the amplification factor $|H(f)|^{-2}$. Taking into account currently available power and bandwidth limitations, an uplink capacity on the order of $1000 \mathrm{bits} / \mathrm{sec}$ can be reached for a drill string approximately $2 \mathrm{~km}$ in length.

\section{DIRECTIONAL SIGNAL ENHANCEMENT}

Considering the fact that the bandwidth, the channel, the signal power and the noise sources are given, one can mistakenly assume that nothing can be done to improve the capacity of the uplink channel. However, we demonstrate here that two receivers can be used to completely remove the surface noise. The key idea is to take the advantage of the fact that compressional acoustic waves travel in two directions inside the drill string. Then, by employing two receivers, surface noise which propagates in the opposite direction from the signal can be perfectly suppressed. Unlike the traditional array processinghere we show that under ideal conditions the two receivers's output can be processed to completely suppress the surface noise.

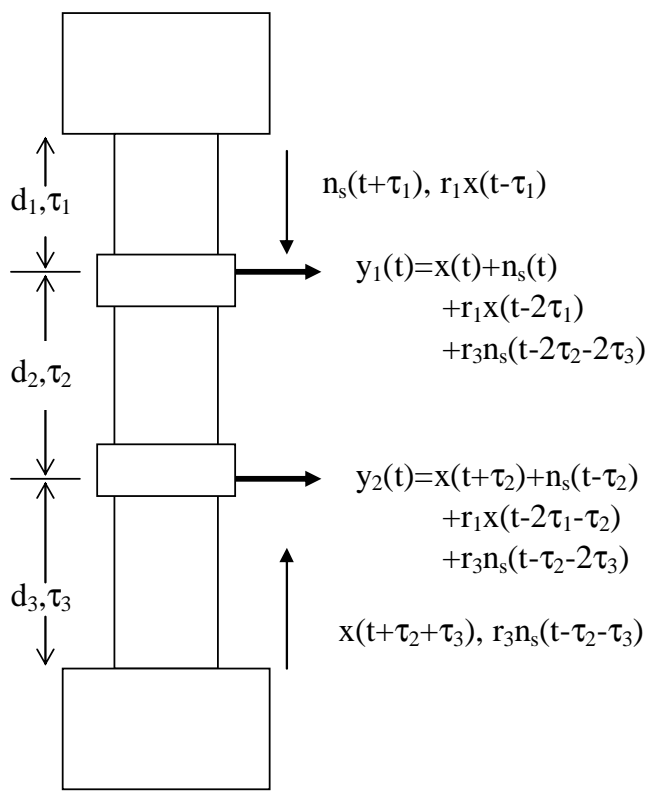

Fig. 2. Two receivers on the first pipe at the top of the drill string record two signals, $n_{s}$ and $x$, and their reflections. Time origin (delay equal to zero) is set at the top receiver. Different delays between the two signals at two receivers can be exploited to completely suppress the surface signal $n_{s}$ and reflections. This case illustrates how directional signal suppression works even with reflections included.

Figure 2 shows the first-order extensional wave reflections at the pipe ends and how they strongly affect what is measured by the two receivers in the frequency domain:

$$
\begin{aligned}
& Y_{1}(f)=H_{X_{1}}(f) X(f)+H_{N_{1}}(f) N_{s}(f) \\
& Y_{2}(f)=H_{X_{2}}(f) X(f)+H_{N_{2}}(f) N_{s}(f)
\end{aligned}
$$

By solving this system for $X(f)$, we get:

$$
X(f)=\left(H_{N_{2}}(f) Y_{1}(f)-H_{N_{1}}(f) Y_{2}(f)\right) / D(f)
$$


where

$$
\begin{aligned}
& H_{X_{1}}(f)=1+r_{1} e^{-j 2 \pi f 2 \tau_{1}} \\
& H_{X_{2}}(f)=e^{+j 2 \pi f \tau_{2}}+r_{1} e^{-j 2 \pi f\left(2 \tau_{1}+\tau_{2}\right)} \\
& H_{N_{1}}(f)=1+r_{3} e^{-j 2 \pi f 2\left(\tau_{2}+\tau_{3}\right)} \\
& H_{N_{2}}(f)=e^{-j 2 \pi f \tau_{2}}+r_{3} e^{-j 2 \pi f\left(\tau_{2}+2 \tau_{3}\right)} \\
& D(f)=H_{X_{1}}(f) H_{N_{2}}(f)-H_{X_{2}}(f) H_{N_{1}}(f)
\end{aligned}
$$

Figure 3 shows that when the acoustic propagation characteristics are well-modeled, the received signal-to-noise ratio can be dramatically enhanced. Since $D(f)$ is a frequency response, as long as it is well-behaved, it does not affect capacity since it filters both the signal and the downhole noise in $X(f)$. Surface noise cancellation significantly improves the uplink capacity, as can be seen from the expression (1) by removing $N_{s}(f)|H(f)|^{-2}$ from the denominator. In our previous work, we considered implementation issues, such as the effects of sampling and quantization on our two-receiver processing [5].

\subsection{Case of Unknown Channel: Training Approach}

Our noise-cancellation scheme presumes perfect knowledge of the channels, which is usually not the case in practice. One approach to tackle the problem of the unknown channels is to use channel modeling. However, modeling is too difficult to achieve in practice due to measurement imprecisions and many parameters involved, such as speed of propagation, sources of wave reflection at pipe joints, different pipe lengths, tools within pipes which complicate the modeling of wave reflection and propagation, etc. We have shown that the cancellation is particularly sensitive to the delay parameters, much more so than uncertainties in the reflection coefficients. Even if the model approaches realworld set-up, it has to be updated constantly since drilling introduces time variations in the model. Therefore, a more practical, adaptive approach is needed.

Training can be used to determine transfer functions used in our scheme. When no signal is coming from the down-hole, we can show that we have all the necessary information to process the two receiver's outputs to cancel the surface noise. Rewriting equation (4), we get:

$$
Q(f) X(f)=\frac{H_{N_{2}}(f)}{H_{N_{1}}(f)} Y_{1}(f)-Y_{2}(f)
$$

where

$$
Q(f)=\frac{H_{N_{2}}(f)}{H_{N_{1}}(f)} H_{X_{1}}(f)-H_{X_{2}}(f)
$$

Note that by using the notation from (6), $Q(f)=$ $D(f) / H_{N_{1}}(f)$. If the ratio $H_{N_{2}}(f) / H_{N_{1}}(f)$ could be obtained, the signal propagating up the drill string and filtered by $Q(f)$ could be calculated from receiver measurements.

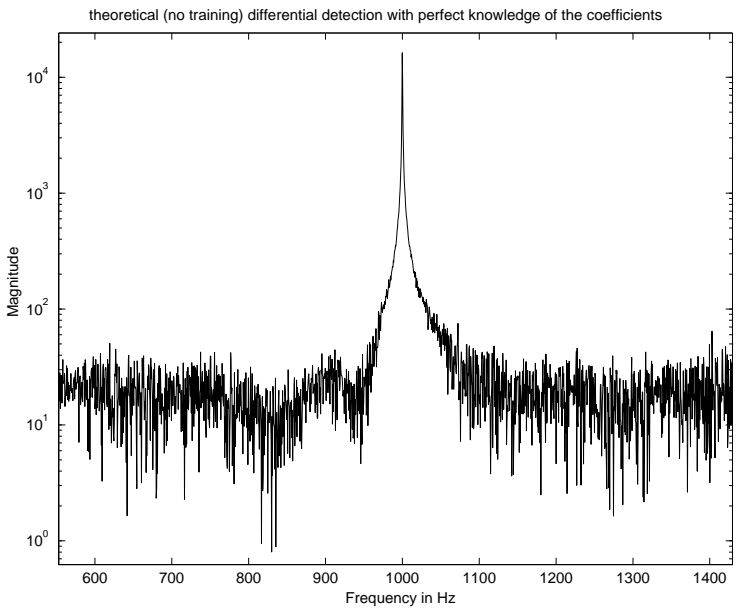

Fig. 3. By assuming perfect knowledge of the coefficients involved in the acoustic telemetry and by using our 2 -receiver processing, we can clearly extract a downhole $1 \mathrm{kHz}$ sine wave from the surface noise, as seen in this frequency-domain plot.

As long as $Q(f)$ is not zero, channel capacity is not affected and, for narrowband data transmission, it need not be known to implement the optimal matched filter receiver. By sending the training signal from the top, while the down-hole signal is absent, the ratio can be found from the training data obtained from the received training signals:

$$
K(f)=Y_{T_{2}}(f) / Y_{T_{1}}(f)=H_{N_{2}}(f) / H_{N_{1}}(f)
$$

for $X(f)=0$, where subscript $T$ 's denote the training data, obtained from the broadband noise propagating from the top. We can now express (7) as

$$
Q(f) X(f)=K(f) Y_{1}(f)-Y_{2}(f)
$$

For $Q(f)$ to be nonzero, we need

$$
\frac{H_{N_{2}}(f)}{H_{N_{1}}(f)} \neq \frac{H_{X_{2}}(f)}{H_{X_{1}}(f)}
$$

\subsection{Imperfect Training}

For what we call perfect training, we assume no signal is propagating up the drill string from downhole sources. This way, we ensure proper calculations of the coefficients needed for processing. However, in drilling applications, some residual downhole noise may be present during training. Specifically, suppose that condition $X(f)=0$ is not valid during the training: there is a residual signal $R(f)$ left and it is coming from the down-hole. The training step can be then described as:

$$
K(f)=\frac{H_{X_{2}}(f) R(f)+H_{N_{2}}(f) N_{T_{s}}(f)}{H_{X_{1}}(f) R(f)+H_{N_{1}}(f) N_{T_{s}}(f)}
$$




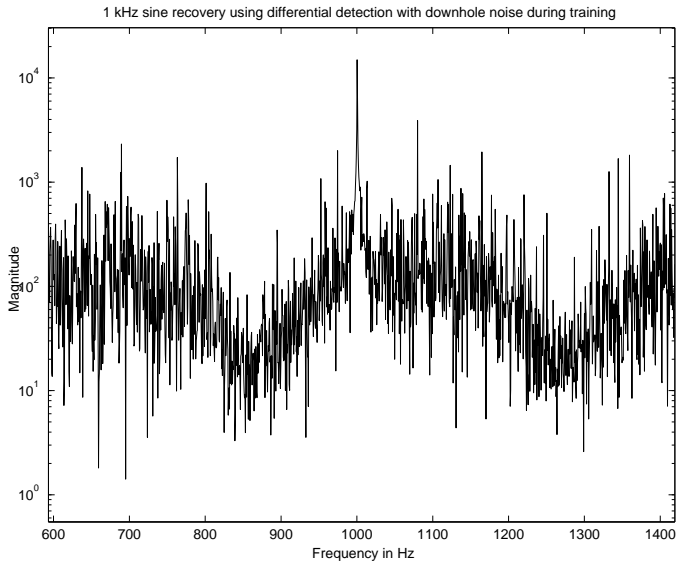

Fig. 4. In the case of imperfect training, downhole residual signal is present as opposed to the perfect training case during which there is no downhole signal. Negative impact on the recovery of $1 \mathrm{kHz}$ sine wave from the noise can be seen on this frequency domain plot. Spikes other than 1 $\mathrm{kHz}$ are prominent and numerous. Performance of the imperfect training is obviously worse than the performance of the perfect training. Sine-wave-to-residual-noise ratio is 2 .

When compared with perfect training (9), last expression shows that the imperfect training introduces the down-hole residual signal and the surface noise in the ratio of the two training sets needed in our compensation filter. Our tworeceiver scheme then becomes:

$$
K(f) Y_{1}(f)-Y_{2}(f)=A(f)\left(X(f)-\frac{R(f)}{N_{T_{s}}(f)} N_{s}(f)\right)
$$

where

$$
A(f)=N_{T_{s}}(f) \frac{H_{N_{2}}(f) H_{X_{1}}(f)-H_{N_{1}}(f) H_{X_{2}}(f)}{H_{X_{1}}(f) R(f)+H_{N_{1}}(f) N_{T_{s}}(f)}
$$

We note that the performance of the two-receiver scheme deteriorates with the presence of the down-hole noise during the training. Simulation confirms and quantifies this phenomenon. See Figure 4. While in the perfect training case (10) downhole signal is perfectly recovered, in the imperfect training case two-receiver scheme introduces noise. We also note that for $R(f)=0$ right-hand side of (13) becomes $Q(f) X(f)$. In other words, imperfect training simplifies to perfect training as in (10). Uplink capacity for the imperfect training is:

$$
C=\int_{B} \log _{2}\left(1+\frac{P_{S}(f)}{P_{N_{b}}(f)+P_{N_{s}}(f)\left|\frac{R(f)}{N_{T_{s}}(f) H(f)}\right|^{2}}\right) d f
$$

\section{CONCLUSIONS}

This paper analyzes performance of a new method of wireless telemetry for oil well service applications that uses compressional acoustic waves to transmit data along the drill string. Acoustic telemetry is capable of achieving data rates on the order of several hundred bits per second but suffers from severe attenuation in the channel, which results in the surface noise dominating the capacity. By using two receivers to suppress the surface noise, the received signal-to-noise ratio (SNR) can be significantly improved resulting in data rates orders of magnitude higher. We have shown that the direction-of-propagation can be exploited to improve channel capacity. This result resembles what has been achieved in MIMO wireless applications. Our approach differs in that we explicitly determine how the two receiver's outputs should be processed and what essential information is needed: the ratio of transfer functions associated with downward propagating signals. Our approach can be extended to more sensors, but in the perfect training case, no more are needed. More sensors could prove useful in the imperfect case, but little of the drill pipe protrudes above the surface and we cannot physically separate them enough to obtain significant performance enhancements.

\section{REFERENCES}

[1] D. S. Drumheller, Acoustical properties of drill strings, J. Acoust. Soc. Am. 85, 1989, pp. 1048-1064.

[2] D.H. Johnson and D.E. Dudgeon, Array Signal Processing: Concepts and Techniques, Prentice-Hall, Inc., 1993.

[3] E. A. Lee and D. G. Messerschmitt, Digital Communication, Kluwer Academic Publishers, 2000.

[4] J. G. Proakis, Digital Communications, McGraw-Hill, 2001.

[5] S. Sinanović, D. Johnson, V. Shah and W. Gardner, Data communication along the drill string using acoustic waves, ICASSP 2004, Montreal, Quebec, Canada, May 17-21, 2004. 